\title{
MEDIDAS DE BIOSEGURIDAD. PROBLEMÁTICA EN RELACION CON LA POBLACION ESTUDIANTIL DEL SECTOR SALUD
}

\section{Sr. Editor:}

En la actualidad se evidencia una disociación entre el conocimiento de bioseguridad y la puesta en práctica de estos conocimientos, como consecuencia se eleva la probabilidad de adquirir enfermedades infecto contagiosas por contacto con secreción contaminadas, manejo de agujas, administración de inyectables, extracción de sangre, reencapuchamiento de agujas, manejos de desperdicios, etc.

Los profesionales de la salud tienen mayor riesgo de sufrir accidentes laborales, sin embargo debido a la inexperiencia y el escaso desarrollo de las habilidades manuales, los estudiantes de pregrado de esta área, son la población en la que se debe mejorar los esfuerzos para optimizar las buenas prácticas en bioseguridad.

Flores Seña, C. (1). en su investigación al respecto observa que existe una relación entre la nota aprobatoria en actitudes y la ocurrencia previa de un incidente ocupacional corto punzante, situación que está alejada del escenario en el que se desenvuelven los estudiantes que recién ingresan al ambiente hospitalario, de este modo esta población se convierte en la más susceptible al riesgo laboral. En nuestro país, los accidentes biológicos en estudiantes de medicina se presentan en un $47 \%$ (2).

En referencia a la investigación titulada "Medidas de bioseguridad que aplica el profesional de enfermería y su relación con la exposición al riesgo laboral en el Hospital Santa María del Socorro. Año 2013 - 2014" (3), relevante evidencia de la frecuencia de la exposición biológica, que en nuestra opinión brinda una idea del panorama que afronta o afrontara la población estudiantil. Respecto a la calificación de las practicas una gran parte de la población estudiada afirmo tener buenas practicas sobre bioseguridad, por citar un resultado el $97,74 \%$ en el ítem: toma de medidas de precaución estándar respecto al lavado de manos siempre, pero se tiene la limitación de no poder verificar presencialmente este hecho, por lo que el resultado puede ser mejorado. Similar resultado tuvo el estudio de Acevedo Iriarte, K. y colaboradores en donde un alto porcentaje (100\%) afirma que aplica las medidas de bioseguridad, pero tampoco se pudo verificar esta acción (4). Somos conscientes que evaluar observacionalmente una población semejante, es una gran tarea, que requiere mucho esfuerzo, no obstante saltar esta limitación representaría una mejora importantísima, que haría la investigación irrefutablemente confiable.

$Y$ es este el sentido, de contribución en el avance del conocimiento de esta problemática con vistas a la toma de medidas que reduzcan los riesgos para los grupos afectados y los estudiantes, población vulnerable, que recomendamos además, extender este trabajo, identificando los incidentes ocupacionales corto punzantes, e investigar la conducta que tomo este grupo cuando se enfrentó a esta circunstancia. De esta forma se conocerá si el personal profesional conoce el protocolo a seguirse en situaciones como esta, y si además de conocerlo, lo llevo a cabo.

Karen Leticia Llerena Ururi. ${ }^{1 a}$

1 Facultad de Medicina, Universidad Nacional de San Agustín. Arequipa, Perú.

Estudiante de Medicina.

Correspondencia:

leticia.llerenau@hotmail.com 


\section{REFERENCIAS BIBLIOGRAFICAS}

1) Flores Seña C, Salmavides Cuba F. Conocimientos sobre bioseguridad en estudiantes de medicina de una universidad peruana. Revista Médica Herediana. Lima oct./dic. 2005. Citado 24 de sep. 2014. Disponible en: http://www.scielo.org.pe/scielo.php?pid=S 1018-130X2005000400005\&script=sci arttext

2) Alva P, Cornejo W, Tapia M, Sevilla C. Medidas de protección contra agentes patógenos transmitidos por sangre, en estudiantes de pregrado. AnFacMed (Lima), 2006; 67(4): 333-348.

3) Soria C. Medidas de bioseguridad que aplica el profesional de enfermería y su relación con la exposición al riesgo laboral en el Hospital Santa María del Socorro. Año 2013 - 2014. Rev. Enferm. Vanguard. 2014; 2(1): 10-16.
4) Acevedo Iriarte $K$, Coha Viloria $K$, Ortiz Martínez A. Evaluación del conocimiento y aplicación de las normas de bioseguridad en estudiantes de enfermería de último año en el servicio de medicina interna de dos IES Cartagena de indias 2013. Grado enfermero profesional, 2013. 137pp

\section{RECIBIDO: $27 / 11 / 14$}

